\title{
GPPS-BJ-2019-0026
}

\section{INCREMENTAL PROPER ORTHOGONAL DECOMPOSITION BASED METHOD FOR THE ANALYZATION OF LARGE SCALE HIGH FIDELITY SIMULATION RESULTS}

\author{
Qingsong Wang \\ Key Laboratory for Thermal Science and \\ Power Engineering of Ministry of Education \\ Institute of Gas Turbine, Department of Energy \\ and Power Engineering \\ Tsinghua University, \\ wangqs15@mails.tsinghua.edu.cn \\ Beijing 100084, P.R. China
}

\author{
Xinrong Su \\ Key Laboratory for Thermal Science and \\ Power Engineering of Ministry of Education \\ Institute of Gas Turbine, Department of Energy \\ and Power Engineering \\ Tsinghua University, \\ suxr@mail.tsinghua.edu.cn \\ Beijing 100084, P.R. China
}

\author{
Zhen Zhang \\ Key Laboratory for Thermal Science and \\ Power Engineering of Ministry of Education \\ Institute of Gas Turbine, Department of Energy \\ and Power Engineering \\ Tsinghua University, \\ z-z17@mails.tsinghua.edu.cn \\ Beijing 100084, P.R. China
}

\author{
Xin Yuan \\ Key Laboratory for Thermal Science and \\ Power Engineering of Ministry of Education \\ Institute of Gas Turbine, Department of Energy \\ and Power Engineering \\ Tsinghua University, \\ yuanxin@mail.tsinghua.edu.cn \\ Beijing 100084, P.R. China
}

\begin{abstract}
Proper Orthogonal Decomposition (POD) analysis is widely used in processing large amounts of high-dimensional data in order to obtain low-dimension approximation, in which way we can capture the most typical modals of the phenomena. Based on this, POD is a powerful tool to analyze complex flow fields. However, there are generally only several modes that dominate unsteady factors, so it is wasteful to calculate all of the modes in analyzing large-scale unsteady calculation results. What's more, the number of grid in simulation of gas turbine is generally huge, so a complete POD which needs full storage costs a lot in memory. Based on incremental singular value decomposition (SVD), a fast incremental POD (iPOD) approach is developed. To compute a full SVD by adding vectors, we iterate the update procedure with truncation based on the user-defined rank, in which way, the left and right singular vector matrices as well as the singular value matrix are updated incrementally, resulting in an online algorithm whose memory requirements reduced several levels. In this work, both 40 and 60 modes are adopted in analyzing the large eddy simulation results of shaped hole film cooling to capture the dominated flow structures and further understand the effect on cooling effectiveness. In comparison, the results of iPOD achieve a good agreement with that of exact method at a low computational cost,
\end{abstract}

demonstrating that the iPOD method is appropriate in large scale high fidelity flow fields analysis.

\section{INTRODUCTION}

Due to the complexity of the flow field in the turbine, it is common that researchers obtain a large number of highdimensional data by experiment and computational fluid dynamics (CFD). Those data describe the flow fields in detail, however, the most typical character may be covered by other information as well. Therefore, it is necessary to find some way of processing the high-dimensional data, so that the dominant factor can be captured.

In the analysis, the first step to extract physically important feature is often modal decomposition. There was an overview about modal analysis of fluid flows by Taira et al (Taira et al., 2017). They presented several well-established modal analysis techniques including POD, balanced POD, dynamic mode decomposition (DMD), Koopman analysis, global linear analysis, and resolvent analysis. The first three methods are data-based, and the rests are operator-based. Among these methods, POD can extract modes ranked by energy ratio, and the first mode occupies the most energy, which generally represents the time-averaged character of the fields. Furthermore, the flow field can be reconstructed by the first several modes that are modes with more energy. 
Consequently, a similar low-dimensional dynamics system can be obtained, which can be used for establishing reducedorder models. In that way, the low-dimensional approximation is accomplished, and the most typical modals of the phenomena can be captured, making further analysis more effective and less cost.

As a powerful tool, POD has been used widely in many researches. Lin et al. (Lin et al., 2018) analyzed the flow mechanism of wake behaviours of high-pressure transonic turbine vane using delayed detached eddy simulation (DDES) and POD. They calculated the correlation coefficient between the time-averaged result from DDES and that reconstructed by POD, which is as high as 0.9998 , proving that the POD analysis was reliable. Further analysis indicated that shedding vortex is the dominant unsteady factor. Bidan et al. (Bidan et al., 2013) performed 3D-POD on LES results of an unforced and modulated film-cooling case in the velocity and temperature fields. By analyzing the first four velocity POD modes, they captured counter-rotating vortices pairs (CRVP), which are the most typical structure in the field, and they deduced that the CRVP originated from the legs of hairpin vortices. Also, the phase shift phenomenon in the modes suggested that the convection of the hairpin was the dominant factor in this flow fields. Then they reconstructed the instantaneous flow field with 20 modes, and the maximum error on the turbulent kinetic energy was less than $2 \%$. Cavar and Meyer (Cavar and Meyer, 2012) applied POD analysis to identify the coherent structures on the flow field of turbulent jet in cross flow. Both 2D and 3D POD analysis were conducted. In 2D POD analysis, the results of PIV-based and LES-based were compared and a good agreement was observed. The LES-based POD analysis was extended to 3D, and the results suggested that structures captured by 3D-POD and 2D-POD had a clear connection, as long as the location of planes were chosen correctly in 2D-POD analysis. This was significant because in this way the calculation cost could be reduced a lot and there was one more way to process the PIV experiment results for a full access to capture the information in the results. Nita et al. (Nita et al., 2014) applied 3D-POD to the optimization of film cooling hole, in which way, the number of design parameters can be reduced and thus further CFD can be more effective. Consequently, the time for design and optimize could be shorten. In fluid dynamics, turbulence is a complex issue, and POD was often adopted, such as obtaining low-dimensional approximation (Holmes et al., 1996) and investigating turbulent flows in curved pipes (Kalpakli et al., 2016). Besides fluid flow, POD was also applied in structural vibrations (Cusumano et al., 1994) (Feeny and Kappagantu, 1998), damage detection (Ruotolo and Surace, 1999) and so on.

The decomposition process of POD is based on SVD. For example, a matrix $Y$ consist of $\mathrm{n}$ rows and m columns, and then the SVD is computed, $Y=U \Sigma V^{T}$, where $U$ is an $n \times n$ orthogonal matrix, $V$ is an $m \times m$ orthogonal matrix, and $\Sigma$ is a $n \times m$ matrix with all elements zero except along the diagonal. The diagonal elements of $\Sigma$ consist of the singular values of $Y$, which are nonnegative number $\sigma_{i}$ and arranged by number based on energy consideration (Chatterjee, 2000). The $\sigma_{i}$ can be computed as square root of eignevalues of matrix $K=Y Y^{T}$, which consist of $n$ rows and $n$ columns. Concerning the actual problem, the data may be so large that the dimension $n$ of the phase space will be large as well. Thus, the calculation of POD would cost a lot for the requirement of solving a full matrix $K \in R^{n \times n}$. To solve this problem, a snapshot method was adopted (Pinnau, 2008). The matrix $Y=$ $\left(y\left(t_{1}\right) \ldots y\left(t_{m}\right)\right) \in R^{n \times m}$ was built, and the columns were consisted of snapshots, which were constructed at certain discrete time instances $t_{1}, \cdots, t_{m} \in[0, T]$. Then the eigenvalue problem would become the matrix $K=Y^{T} Y \in$ $R^{m \times m}$, hence, the calculation would cost less for the number of snapshots $m$ was generally much less than the dimension $n$.

However, in the CFD of turbomachinery, as the calculation accuracy is improving, the requirement of grid is getting higher as well. The truth is that the scale of result data has a positive correlation with the fidelity. In this work, the effect of cross flow on film cooling is investigated by Large Eddy Simulation (LES) using subgrid-scale (SGS) model. The total grid cell number is 17.25 million, and most of them are distributed around the cooling hole and downstream to capture the key flow structures. Even though the snapshot method is adopted, the calculation of 3D-POD still cost much, especially memory. What's more, the dense grid in the key zone causes that the rank of the field matrix is far less than the rows due to the tall and thin character of the matrix. Based on Lanczos methods, a fast incremental SVD (Brand, 2002) is adopted since the rank of the field matrix is far less than the rows due to the tall and thin character of the matrix. With the current incremental approach, the POD can be done on-the-fly and every snapshot can be added sequentially, thus avoiding the high requirement of computer memory and cost.

The rest of this paper is organized as follows. The next section introduces iPOD method and LES setup in detail. Then the application of iPOD in this LES is discussed. Finally, the last section concludes the whole paper.

\section{METHODOLOGY}

\section{Incremental singular value decomposition (iSVD)}

Let the matrix $Y=\left(y\left(t_{1}\right) \ldots y\left(t_{m}\right)\right) \in R^{n \times m}$. In CFD, $\mathrm{n}$ is equal to the number of grid, and $m$ is equal to the number of snapshots, that is the number of time instance. By SVD, $Y=$ $U \Sigma V^{T}$, as previous description, the diagonal elements of $\Sigma$ consist of the singular values of $Y$, which are nonnegative number $\sigma_{i}$ and arranged by number based on energy consideration, i.e. $\sigma_{1} \geq \sigma_{2} \geq \cdots \geq \sigma_{r} \geq 0$, for any $d \leq$ $r$, the matrix $\Sigma_{d}$ can be obtained by setting $\sigma_{r+1}=\sigma_{r+2}=$ $\cdots=\sigma_{r}=0$, and then a reduced-dimension approximation can be obtained by $Y_{d}=U_{n \times d} \Sigma_{d \times d} V_{m \times d}^{T}$. However, in this case, due to the dense grid, the matrix $Y$ is tall and thin, which results in $\leq m \leq n$. Therefore, it is wasteful to compute a full SVD. So we adopted a fast incremental SVD of low-rank matrix.

The core of the developed iPOD algorithm is to replace the direct decomposition of all LES data sets with a smallscale matrix SVD decomposition. The iteration process is as following. Based on existing instantaneous fields' decomposition results, that is $Y_{d}=U_{n \times d} \Sigma_{d \times d} V_{m \times d}^{T}$, and then 
the next time step LES result is added as shown in equation (1).

$$
\left[\begin{array}{ll}
U \Sigma V^{T} & \boldsymbol{y}
\end{array}\right]=\left[\begin{array}{ll}
U & \boldsymbol{f}
\end{array}\right]\left[\begin{array}{ll}
\Sigma & \boldsymbol{h} \\
0 & k
\end{array}\right]\left[\begin{array}{ll}
V & 0 \\
0 & 1
\end{array}\right]^{T}
$$

Let $H=\left[\begin{array}{ll}U & \boldsymbol{f}\end{array}\right] \in R^{n \times(d+1)}, Q=\left[\begin{array}{ll}\Sigma & \boldsymbol{h} \\ 0 & k\end{array}\right] \in R^{(d+1) \times(d+1)}$, $J=\left[\begin{array}{ll}V & 0 \\ 0 & 1\end{array}\right] \epsilon R^{(m+1) \times(d+1)}$,

$$
\boldsymbol{h}=U^{T} \boldsymbol{y}
$$

According to equation (1),

$$
\boldsymbol{y}=U \boldsymbol{h}+k \boldsymbol{f}
$$

Let $\|f\|_{L_{2}}=1$, from equation(3),

$$
\begin{aligned}
& k=\left\|\left(I-U U^{T}\right) \boldsymbol{y}\right\|_{L_{2}} \\
& \boldsymbol{f}=\left(I-U U^{T}\right) \boldsymbol{y} / k
\end{aligned}
$$

To update SVD, the middle matrix $Q$ must be diagonalzed as following,

$$
Q=\left[\begin{array}{ll}
\Sigma & \boldsymbol{h} \\
0 & k
\end{array}\right]=U^{\prime} \Sigma^{\prime} V^{\prime T}
$$

where $U^{\prime}, \Sigma^{\prime}, V^{\prime} \in R^{(d+1) \times(d+1)}$.

Then the updated $U, \Sigma, V$ can be replaced by

$$
\begin{aligned}
& U^{\prime \prime}=H U^{\prime} \epsilon R^{n \times(d+1)} \\
& \Sigma^{\prime \prime}=\Sigma^{\prime} \epsilon R^{(d+1) \times(d+1)} \\
& V^{\prime \prime}=J V^{\prime} \in R^{(m+1) \times(d+1)}
\end{aligned}
$$

However, as shown above, the dimension is added, which should not appear, so truncation is performed during the process of update to preserve the dimension $d$ as following:

$$
\begin{aligned}
& U^{\prime \prime} \leftarrow H U_{[:, 1: d]}^{\prime} \\
& \Sigma^{\prime \prime} \leftarrow \Sigma_{[1: d, 1: d]}^{\prime} \\
& V^{\prime \prime} \leftarrow J V_{[1: d, 1: d]}^{\prime}
\end{aligned}
$$

Now, an iterate is completed, and the operation decreased to $O\left((n+m) d^{2}+n\right)$ from $O(n m d)$ compared to standard SVD, which save lots of computation sources for $d \ll m \ll$ $n$. What's more, the results of LES are added one by one instead of reading the all of the data one time, so the high requirement of memory can be avoided as well. It is worth noting that the dimension $d$ is set in advance, so whether the dimension is enough to character the fields accurately needs validation.

\section{LES setup}

As shown in Figure 1 the computational domain is part of a semi-circular channel with a rectangular plenum. The radius of inner wall is $R_{1}=320 \mathrm{~mm}$, and the outer wall is $R_{2}=480 \mathrm{~mm}$. The 7-7-7 cooling hole is located at 35:5 degrees from the inlet and 58:5 degrees from the outlet. The diameter of 7-7-7 shaped hole is $D=4 \mathrm{~mm}$ with the incidence of 30 degrees to the surface. The expasion angle in each direction is $7^{\circ}$. More detailed introduction about 7-7-7 shaped hole is provided by Schroeder and Thole. (Schroeder and Thole, 2014). The height of the geometry is $20 D$, and the plenum is $3 D \times 15 D \times 10 D$ in the $x, y$ and $z$ directions, respectively. The commercial software ANSYS ICEM is adopted to generate the multiblock structured mesh. The total grid cell number is 17.25 million, as shown in Figure 1.

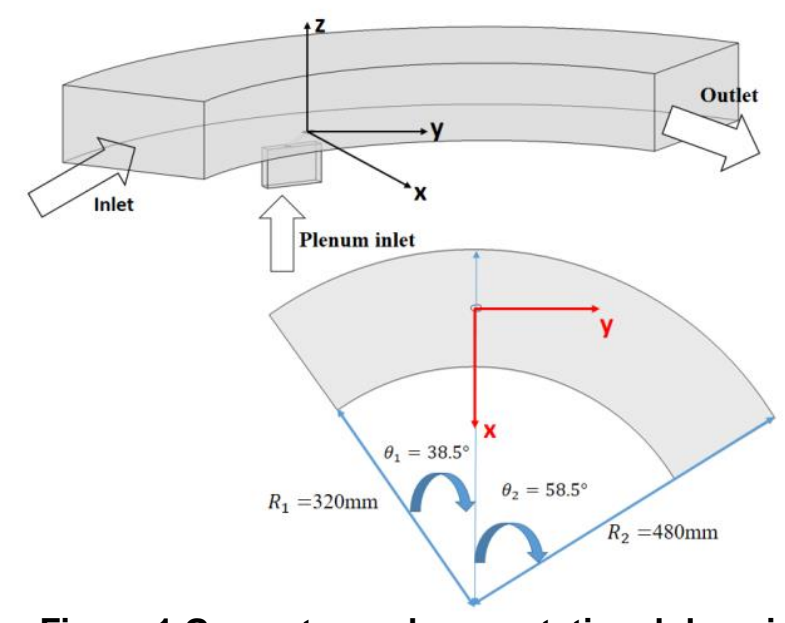

Figure 1 Geometry and computational domain

Most of the grids are distributed around the cooling hole and downstream to capture the key flow structures. In the $z$ direction, the maximum $\Delta z^{+}$from the first layer of the wall meshes is less than 1 . The streamwise-direction is $\Delta y+<40$ and the spanwise direction is $\Delta x+<20$ in the key zone. The commercial software ANSYS FLUENT 16.1 is used in the simulation for LES. Smagorinsky-Lilly model is adopted to compute the SGS viscosity. Computation is performed as incompressible flow condition with ideal-gas air and tempereature-coupled density and viscosity. The momentum term is discretized with bounded central differencing to reduce numerical dissipation and the energy term is discretized with second order upwind scheme. The time discretization is bounded second order implicit. The time step is $4.7 \times 10^{-6} \mathrm{~s}$ to ensure the maximum Courant Friedrichs Lewy (CFL) number is around 0.9 .

The total pressure at the inlet is $102360 \mathrm{~Pa}$ and the inlet total temperature is $300 \mathrm{~K}$ to simulate the velocity of $35 \mathrm{~m} / \mathrm{s}$ in the mainstream, the same as the experiment. To simulate the case of blowing ratio $M=1$, at the inlet of plenum, the inlet velocity is $0.6 \mathrm{~m} / \mathrm{s}$, so the Reynolds number is about 9584 related to the diameter of cooling hole, and the inlet total temperature is $273 \mathrm{~K}$. The symmetrical plane in the mainstream and the side walls of the plenum are regarded as slipped walls, and the other ones are set as adiabatic nonslipped walls.(Li et al., 2017)

\section{Coordinate transformation and key area extraction}

In iPOD analysis, the flow field information in the key area is extracted to analyze rather than the whole computational zone as shown in Figure 2.

Based on the distribution of coolant on the endwall, we extract the key zone that contains 6302698 elements identified by $0.375 \mathrm{~m}<r<0.410 \mathrm{~m}, \quad-0.021<\theta<0.17$ 
and $-0.007 \mathrm{~m}<z<0: 020 \mathrm{~m}$, in which $\mathrm{r}$ and $\theta$ are defined as below:

$$
\begin{aligned}
r & =\sqrt{y^{2}+(x-0.4)^{2}} \\
\theta & =\arctan \left(\frac{y}{0.4-x}\right) \\
X & =0.4-r ; Y=r * \theta ; Z=Z
\end{aligned}
$$

where $0.4 \mathrm{~m}$ is the radius of the film cooling hole location. Correspondingly, the region is limited by

$$
\begin{aligned}
& -2.5<\frac{X}{D}<6.25 \\
& -1.97<\frac{Y}{D}<17.43 \\
& -1.75<Z / D<5
\end{aligned}
$$

under $X, Y, Z$ coordinate system.

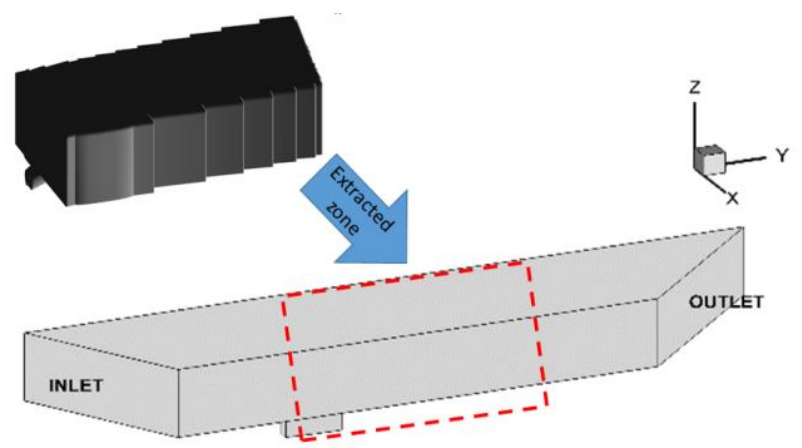

Figure 2 Demonstration of the extracted zone in the $X ; Y ; Z$ coordinate system

\section{RESULTS AND DISCUSSION}

Before analyzing the intantaneous results by iPOD, the accuracy of LES is validated by lateral averaged cooling effectiveness. Figure 3 presents the experiment, LES, and RANS results of lateral averaged cooling effectiveness in the $X ; Y ; Z$ coordinate system, in which we find that the LES is consistent with the experiment and RANS is over-predicted in contrast to LES.

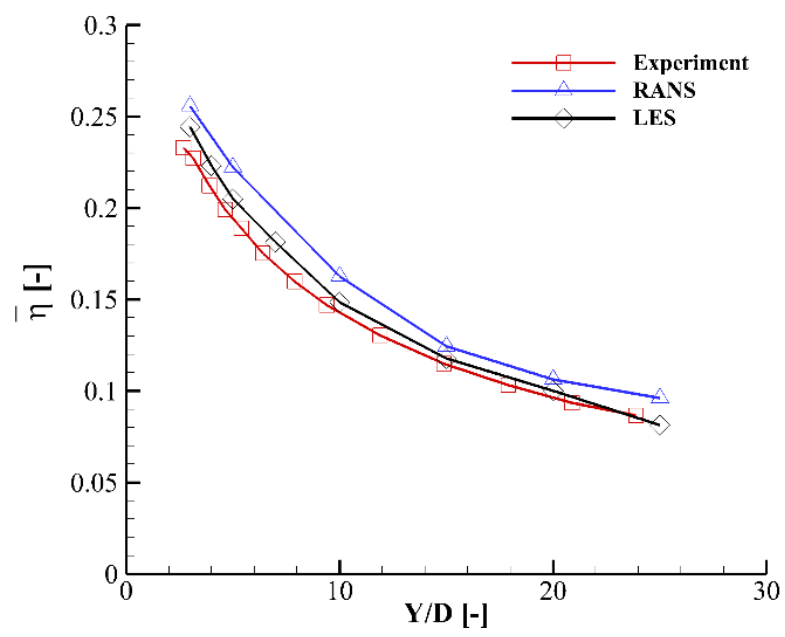

Figure 3 Lateral average cooling effectiveness in the $X ; Y ; Z$ coordinate system
To capture the flow modes of the fields, initial $x$-direction velocity $(u), y$-direction velocity $(v), z$-direction velocity $(w)$ and temperature are chosen as the analyzed variables. To ensure the dimension $\mathrm{d}$ chosen is proper, 40 modes and 60 modes for 108 snapshots are separately chosen to compare. The relevant coefficient between mode 0 of two results is as high as 0.9997 according to equation (19), where $u_{0}^{40}$ and $u_{0}^{60}$ are the $0^{\text {th }}$ mode obtained by iPOD output 40 modes and 60 modes separately, and 6302698 is the number of grid number, $i$ represents the variable number. Figure 4 shows eigenvalue proportion for all of the modes. Except for mode 0 , the eigenvalue proportion for mode 1-40 is similar, which means the iPOD analysis convergence, so we adopt 40 modes to reduce calculation costs. Then we analyze the eignevalues of 40 modes except the 0th mode in the detail show of Figure 4. We find that except the first mode, the eignevalues of other 39 modes gradually decrease and the decline in eigenvalues of mode 1-5 is obviously in contrast to others. In fact, the first 6 modes singular value proportion sum up to $90.62 \%$, which means that the first 6 modes include most energy of the fields and the dominant unsteady factors are included in mode 1 to mode 5 , so these modes are analyzed in detail later.

$$
R=\frac{\sum_{1}^{6302698 \times 4} u_{0 i}^{40} \times u_{0 i}^{60}}{\left\|u_{0}^{40}\right\|_{L_{2}}\left\|u_{0}^{60}\right\|_{L_{2}}}=\sum_{1}^{6302698} u_{0 i}^{40} \times u_{0 i}^{60}
$$

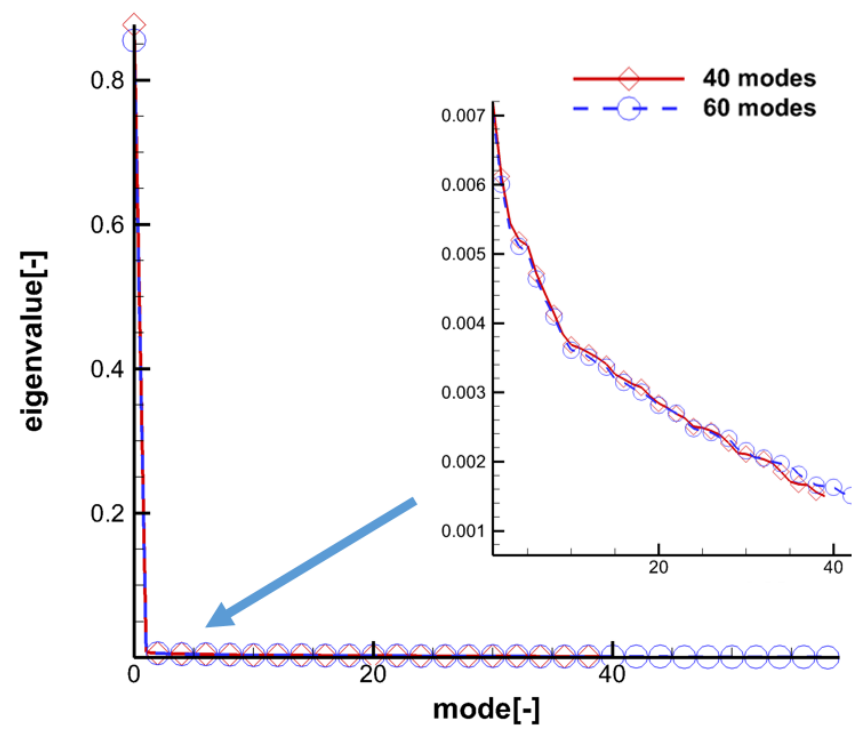

Figure 4 Eignevalue proportion of modes.

The 0th mode with the biggest eignevalues represents the time-averaged fields which shows the most typical features of jet in the cross flow. In the streamwise velocity contour, we can clearly identify the separation and acceleration zone in the diffusion section of cooling hole from mode 0 in Figure 5a. In contrast to the time-averaged result in Figure $5 b$ from LES, a good agreement is obtained, and some typical character is captured in mode 0 . By the way, in the contour of mode 0 , the absolute value of $v$ doesn't equal to the true value, for the mode has been normalized, and the true value can be obtained by multiplying the singular value related to mode 0 . 


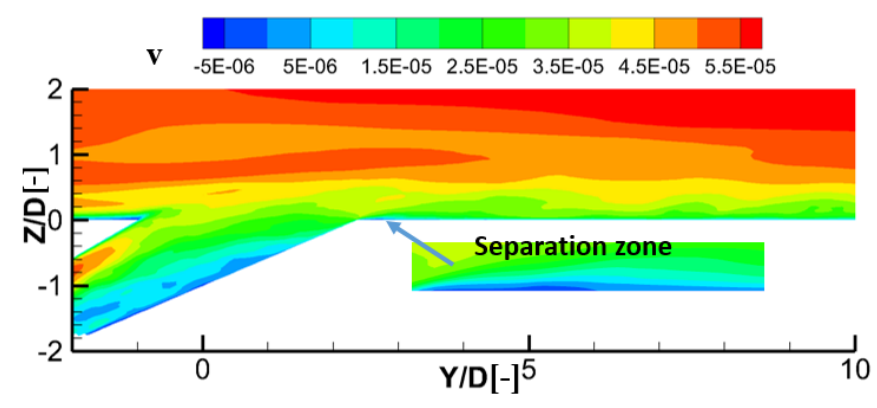

a) mode 0

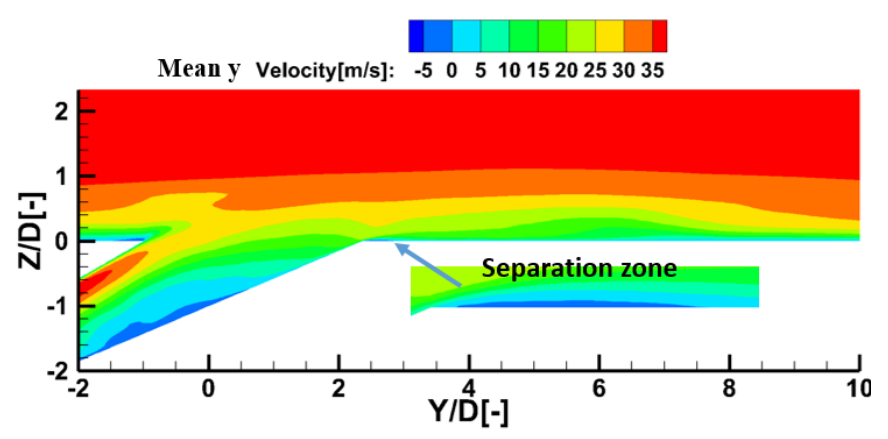

b) time-averaged result by LES

Figure 5 The distribution of $y$-velocity on the center plane of mode 0 and time-averaged result by LES

At the exit of the cooling hole $(Y / D=1,2$ and 3$)$, the spatial distribution of temperature is quite similar to the results of time-averaged field as well. What's more, the asymmetry of low temperature zone which is owing to cross flow is quite obvious. It can be deduced that the symmetry of coherent vortices is damaged by cross flow. In fact, from instantaneous field result of LES, the counter rotating vortices pairs are changed by cross flow. The vortices with negative vorticity are forced to the endwall. On the contrary, those with positive vorticity are lifted, that's why the temperature distribution shows asymmetry. All above is the information we get from mode 0 , which helps us capture the most typical character of the fields and guide the further analysis in detail during the huge amount of information provided by LES.
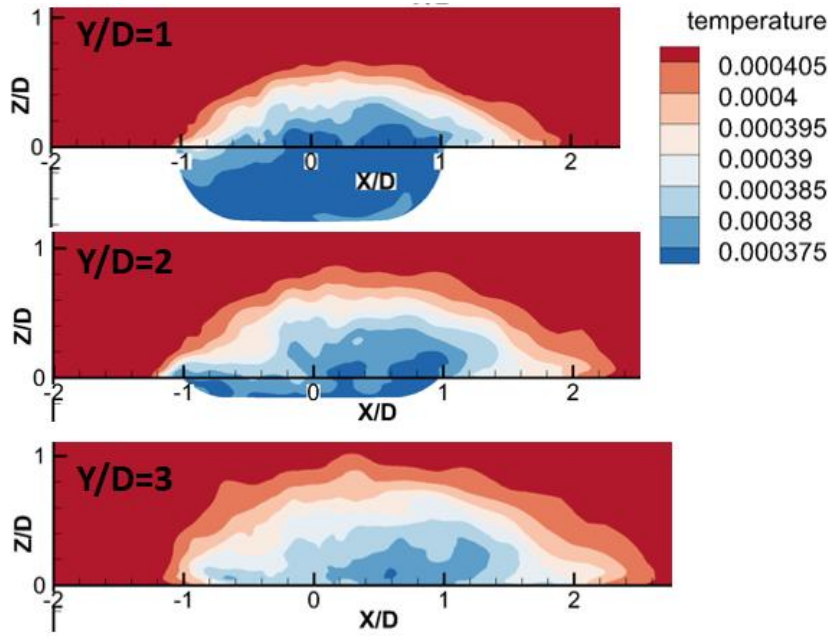

\section{Figure 6 The distribution of temperature at cross section view of mode 0}

The distribution of temperature and y-velocity on the center plane of mode 1-2 are presented in Figure $7 \mathrm{a}$ and Figure $7 \mathrm{~b}$. The $1 \mathrm{st}$ and 2 nd modes characterize the interaction between coolant and mainstream, according to the contour. What's more, according to the velocity contour, there are large scale vortices in the mainstream, which has been confirmed by the instantaneous vortices identified by $\mathrm{Q}$ criterion iso-surface in figure 10 .
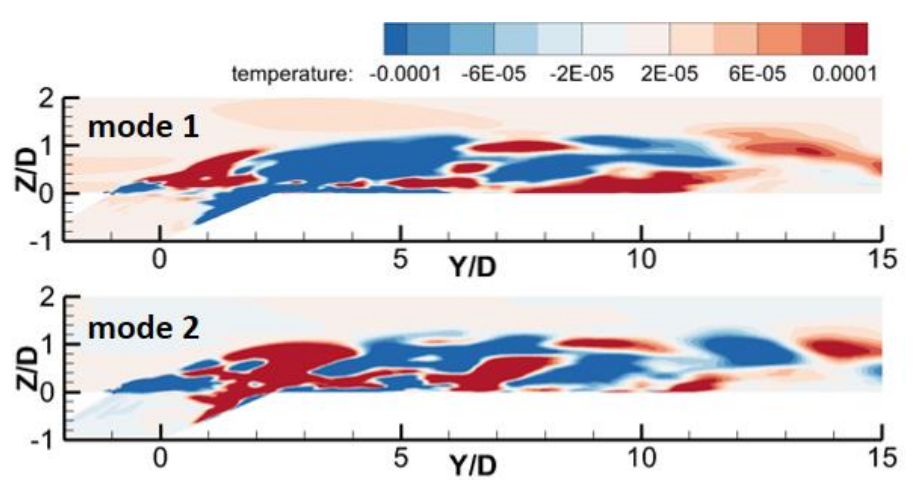

a) The distribution of temperature
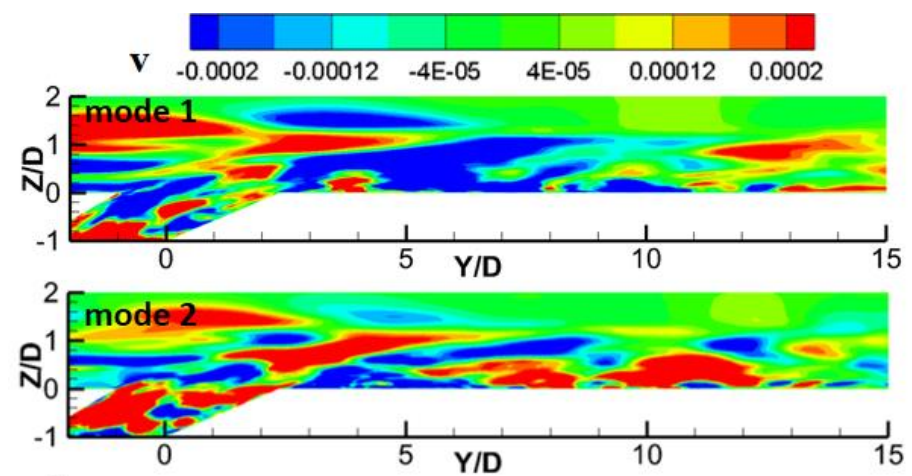

b) The distribution of $y$-velocity

Figure 7 The distribution of temperature and $y$ velocity on the center plane of mode 1-2 
While comparing the temperature and velocity contour at the same location of 3rd, 4th and 5th, it can be found that they resemble each other with a phase shift, especially in the temperature contour. In the velocity contour, the different direction streamwise velocity component appear in turn. The behaviour is associated with the convection of hairpin vortices in the shear layer, which has been observed in the instantaneous fields in figure 10 as a typical flow structure in this case.
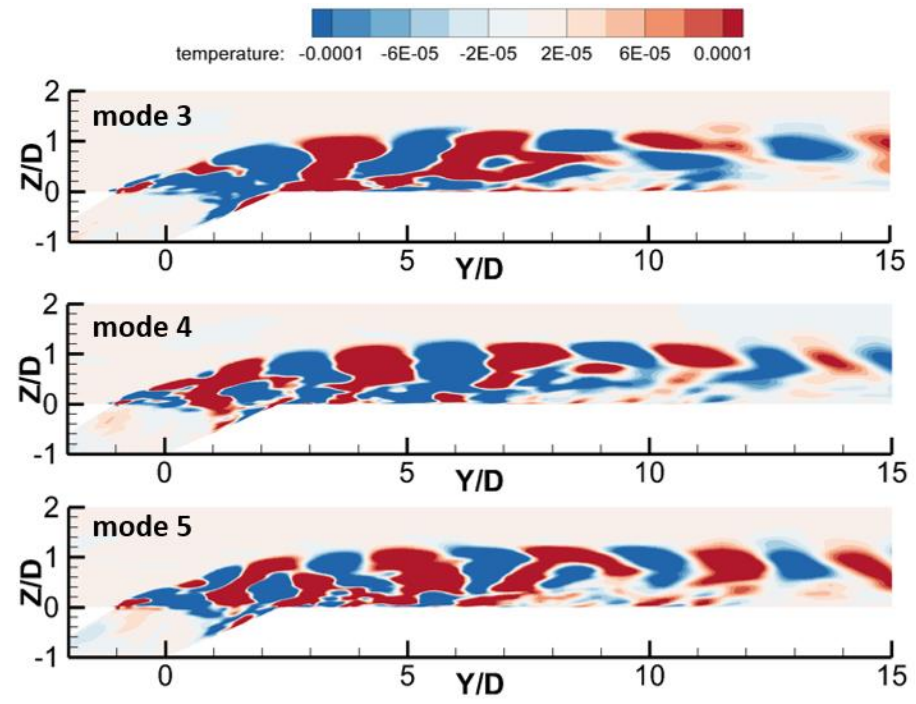

a) The distribution of temperature
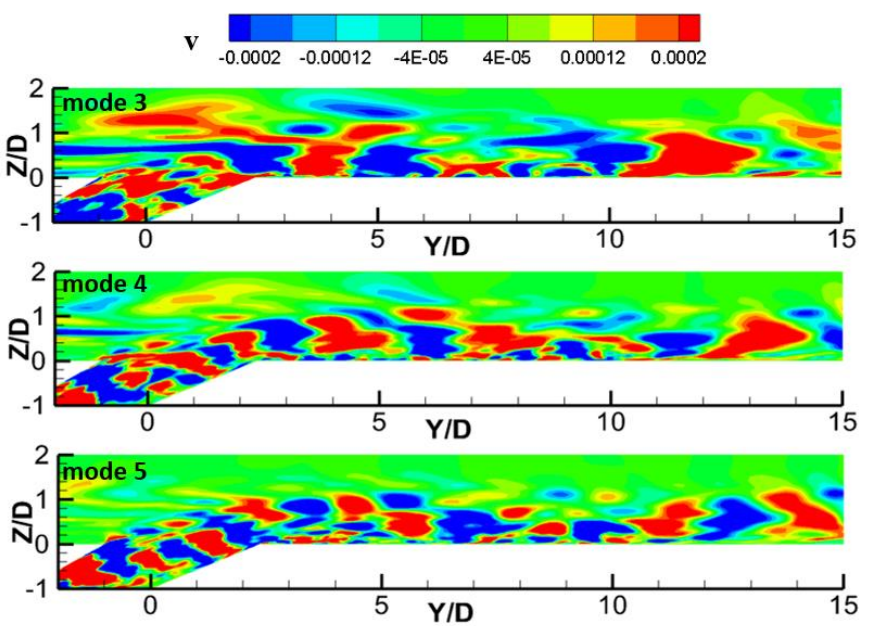

b) The distribution of $y$-velocity

Figure 8 The distribution of temperature and $y$ velocity on the center plane of mode 3-5

Correspondingly, the contour of $(Y / D=1,2$ and 3) plane of 4th mode in Figure 9 shows similar shapes of hairpin vortices at the exit of the cooling hole, so 3rd, 4th and 5th mode represent the convection of hairpin vortices. What's more, due to the influence of cross flow, the vortices on the upwind push down the vortices on the downwind, which causes the vortices on the downwind to gradually dissipate. As is shown in Figure 4, the 6th mode as well as other modes with relatively small eignevalues $(\mathrm{N}>5)$ mainly describe near-wall flow fields especially the interaction between small-scale vortices and hairpin vortices.
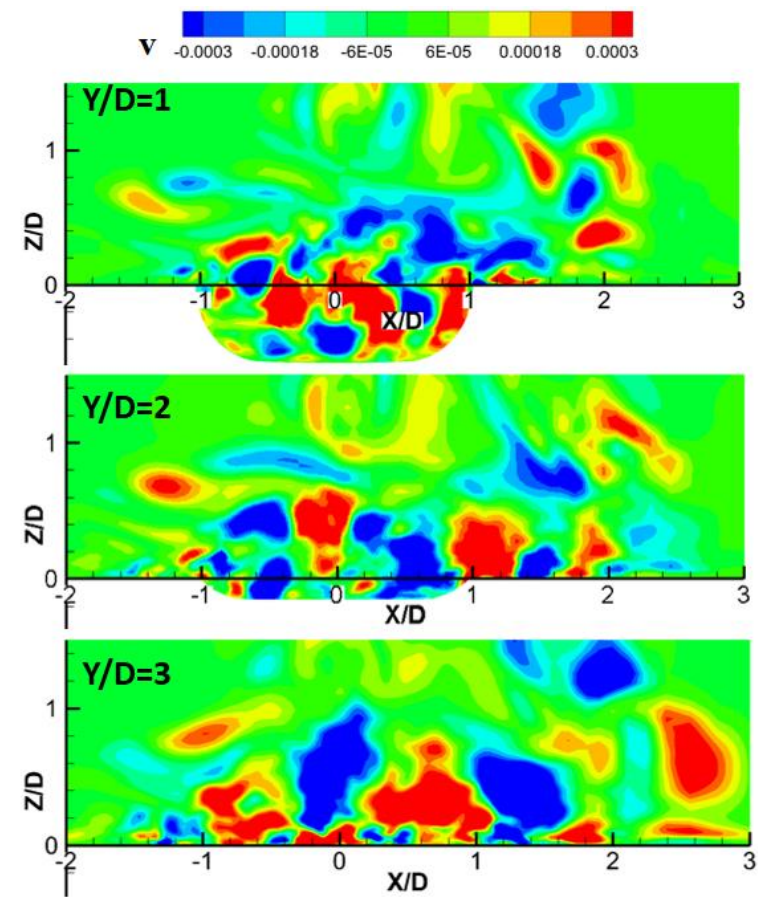

\section{Figure 9 The distribution of $y$-velocity at the cross section view $(Y / D=1,2$ and 3 ) of mode 4}

With the help of iPOD, we can further analyze the intantaneous field in detail. It is easy to find that there are large-scale vortices generating from the mainstream upstream the cooling hole and many hairpin vortices formed at the exit of the shaped hole in Figure 10. It is worth noting that the origin results are disorganized due to the large amounts of information, and it is the iPOD that makes the analyzation more effective.

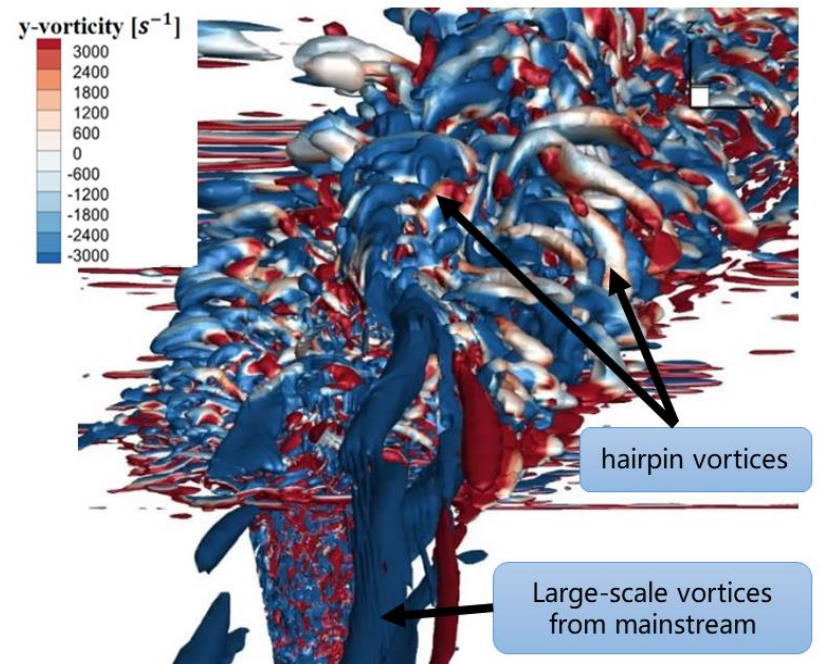

Figure 10 Instantaneous vortices identified by $Q=0.09$ iso-surface 


\section{CONCLUSIONS}

In this work, a fast incremental POD (iPOD) approach is developed to analyze the complex field provided by LES. The algorithm of iSVD is introduced in detail at first, which reduces the expense of computation by iteration with truncation.

To ensure the analysis convergence, both 40 and 60 modes are outputted. By computing the relevant coefficient of mode 0 and comparing the singular value, a good convergence is obtained. What's more, the agreement between mode 0 of 40 modes and the time-averaged field of LES result confirms that.

To analyze the influence mechanism of cross flow on shaped hole film cooling, the 0th mode, which occupies the most energy is analyzed first. The asymmetry of temperature distribution suggests that coherent vortices may be changed by cross flow, which is confirmed by the instantaneous vortices identified by $\mathrm{Q}$ criterion iso-surface. And then in order to analyze the unsteady factor, mode 1-5 are discussed. Combined with instantaneous fields, the large scale vortices from mainstream and the convection of hairpin vortices in the shear layer are discovered as the dominant unsteady factor.

To sum it up, this paper provided a new tool to process a large amounts of high-dimension data, which is common in the research of turbomachinery. The proper method can make full use of large scale high fidelity simulation results.

\section{NOMENCLATURE}

$D$ diameter of the cooling hole, $\mathrm{mm}$

$x \quad$ Cartesian coordinate system (Lateral)

$y \quad$ Cartesian coordinate system (Streamwise)

$z \quad$ Cartesian coordinate system (Normal of the endwall)

$M$ blowing ratio

$v \quad$ the velocity in the $\mathrm{y}$ direction

$r \quad$ cylindrical coordinate (Radial)

$\theta \quad$ cylindrical coordinate (Circumferential)

$X \quad X ; Y ; Z$ coordinate system (Lateral)

$Y \quad X ; Y ; Z$ coordinate system (Streamwise)

$Z \quad X ; Y ; Z$ coordinate system (Normal of the endwall)

SVD Singular Value Decomposition

POD Proper Orthogonal Decomposition

iPOD incremental POD

CRVP counter-rotating vortex pair

\section{ACKNOWLEDGMENTS}

This study is supported by the National Natural Science Foundation of China (Project Grant No. 51876098) and National Science and Technology Major Project (2017-III0009-0035), also supported by the Open fund from State Key Laboratory of Aerodynamics.

\section{References}

Bidan G., Vézier C., Nikitopoulos D.E.. Study of Unforced and Modulated Film-Cooling Jets Using Proper Orthogonal Decomposition-Part I: Unforced Jets. ASME. Journal of Turbomachery. 2012; 135(2):021037-021037-11. doi: $10.1115 / 1.4006599$.
Brand M.. Incremental singular value decomposition of uncertain data with missing values. 7th European Conference on Computer Vision (ECCV 2002). Springer, 2002.

Cavar, D., and Meyer, K. E. (2012). LES of turbulent jet in cross flow: Part 2 - pod analysis and identification of coherent structures. International Journal of Heat and Fluid Flow, 36, 35 - 46. doi:10.1016/j.ijheatfluidflow.2012.03.010

Chatterjee, A. (2000). An introduction to the proper orthogonal decomposition. Current Science, 78(7), 171-4.

Cusumano, J. P., Sharkady, M. T. and Kimble, B. W., Philos. Trans. R. Soc. London, Ser. A. (1994), 347, 421-438.

Feeny, B. F. and Kappagantu, R., J. Sound Vibr.(1998) 211, 607-616.

Holmes, P., Lumley, J. L. and Berkooz, G.(1996). Turbulence, Coherent Structures, Dynamical Systems and Symmetry, Cambridge Monogr. Mech., Cambridge University Press,

Kalpakli Vester, A., Örlü, R., and Alfredsson, P. H.(2016). Turbulent flows in curved pipes: Recent advances in experiments and simulations. Applied Mechanics Reviews, 68(5). doi:10.1115/1.4034135

Li Y., Zhang Y., Su X., Yuan X.. Influence of Mainstream Cross Flow on Film Cooling Performance and Jet Flow Field.(2017) ASME. Turbo Expo: Power for Land, Sea, and Air, Volume 5A: Heat Transfer ():V05AT12A013. doi:10.1115/GT2017-64234.

Lin D., Su X., Yuan X.(2018). The Development and Mechanisms of the High Pressure Turbine Vane Wake Vortex. ASME. J. Eng. Gas Turbines Power.140(9):092601-09260113. doi:10.1115/1.4039802.

Nita, K., Okita, Y., Kubo, S., Yonekura, K., and Watanabe, O.(2014). Film cooling hole shape optimization using proper orthogonal decomposition . In ASME. Turbo Expo: Power for Land, Sea, and Air, Vol. 2B: Turbomachinery: V02BT39A046. doi: 10.1115/GT2014-27239

Pinnau, R.(2008) . Model reduction via proper orthogonal decomposition. Springer Berlin Heidelberg

Schroeder RP, Thole KA. Adiabatic Effectiveness Measurements for a Baseline Shaped Film Cooling Hole. ASME. Turbo Expo: Power for Land, Sea, and Air, Volume 5B: Heat Transfer ():V05BT13A036. doi:10.1115/GT201425992.

Taira, K., Brunton, S. L., Dawson, S. T. M., Rowley, C.W.,Colonius, T., Mckeon, B. J., Schmidt, O. T., Gordeyev, S., Theofilis, V., and Ukeiley, L. S.(2017). Modal analysis of fluid flows: An overview. American Institute of Aeronautics and Astronautics Journal, 55(12). doi: 10.2514/1.j056060

Ruotolo, R. and Surace, C., J. (1999) Sound Vibr. 226, 425439. 